\title{
๖Tropical Cyclone Center Fix Using CYGNSS Winds
}

\author{
DAVID MAYERS AND CHRISTOPHER RUF
}

University of Michigan, Ann Arbor, Michigan

(Manuscript received 4 March 2019, in final form 10 June 2019)

\begin{abstract}
A new method is described for determining the center location of a tropical cyclone (TC) using wind speed measurements by the NASA Cyclone Global Navigation Satellite System (CYGNSS). CYGNSS measurements made during TC overpasses are used to constrain a parametric wind speed model in which storm center location is varied. The "MTrack" storm center location is selected to minimize the residual difference between model and measurement. Results of the MTrack center fix are compared to the National Hurricane Center (NHC) Best Track, the Automated Rotational Center Hurricane Eye Retrieval (ARCHER), and aircraft reconnaissance fixes for category 1-category 3 TCs during the 2017 and 2018 hurricane seasons. MTrack produces storm center locations at intermediate times between NHC fixes with a factor of 5.6 overall reduction in sensitivity to uncertainties in the NHC fixes between which it interpolates. The MTrack uncertainty is found to be larger in the cross-track direction than the along-track direction, although this behavior and the absolute accuracy of position estimates require further investigation.
\end{abstract}

\section{Introduction}

Locating the storm center of a tropical cyclone (TC) is critical for forecasting and analysis. The Dvorak intensity estimation technique, one of the more commonly used methods for monitoring TCs, requires a storm center fix as its first step (Olander and Velden 2007). Methods that attempt to predict rapid intensification also require the location of the storm center (Rozoff et al. 2015; Kieper and Jiang 2012). It is no exaggeration to say that all TC simulation studies utilize the storm center location as an input, so the fix is very important for reanalysis. For all its importance, storm centers can vary between different World Meteorological Organization (WMO) regional centers as indicated by the WMO IBTrACS archive (Knapp et al. 2010; Kruk et al. 2010). Often, TC center and intensity estimation is performed manually by experienced forecasters rather than by an automated computer algorithm, such as the Automated Rotational Center Hurricane Eye Retrieval (ARCHER; Wimmers and Velden 2010, 2016), which could perform with more consistency and less subjectivity.

Denotes content that is immediately available upon publication as open access.

Corresponding author: David Mayers, drmayers@umich.edu
Most current techniques fit expected TC shapes to satellite imagery to pinpoint the center. A common technique is to fit spiral or ring shapes to visible, infrared, or passive microwave satellite imagery of the TC (Wimmers and Velden 2010; Bai et al. 2012). These techniques perform best for well-organized storms with a clear eye. The spiral shape is matched to the rainbands of the TC, but this can be difficult for disorganized storms with poorly defined bands. The ring fitting technique attempts to find the eyewall, but obviously this requires the presence of an eye, which occurs more often in intense storms with good organization. Ring fitting also has the potential to identify false eyes away from the center of the storm. Both ring and spiral fitting methods are limited by the organization of the storm and lack of visible satellite imagery at night. They are also restricted to only the $12.6 \%$ of cases that have a detectable eye in satellite imagery (Knapp et al. 2018).

Another method is to use wind vectors from scatterometers that can be traced inward to the storm center, but the quality of wind speed and direction is known to degrade in heavy precipitation. This method is most often used for weaker storms with no clear eye that do not have an accurate fix by spiral or ring fitting. Scatterometers have more directional ambiguity at the lower winds that occur in weaker storms, however, so this is 


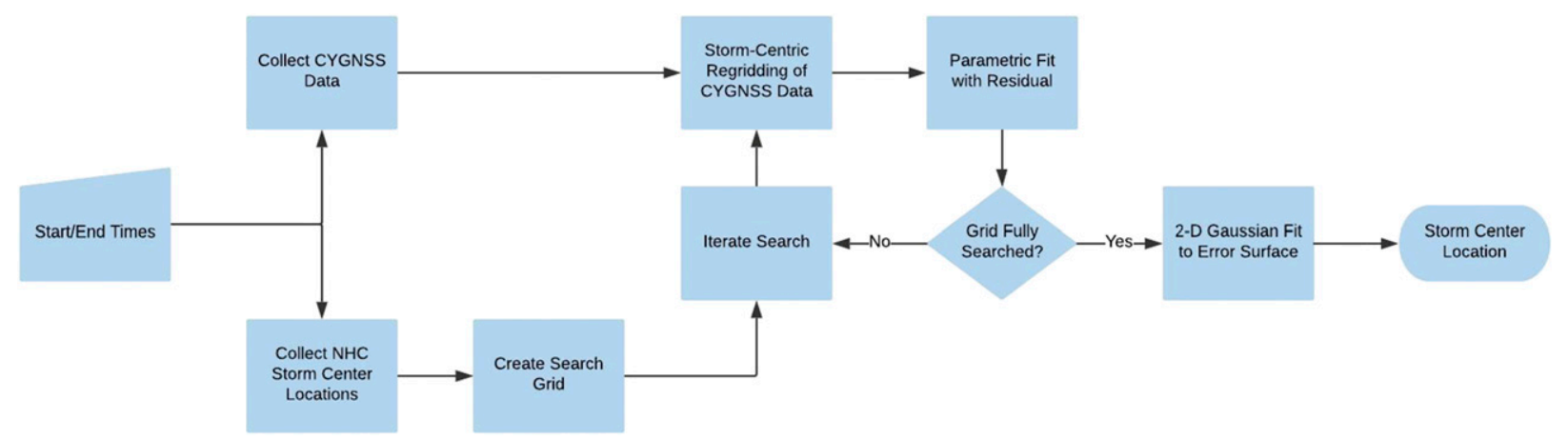

FIG. 1. Flowchart of the MTrack storm center-finding algorithm. First, CYGNSS surface wind speed data and NHC Best Track fixes are collected for the selected time interval. A search grid centered on the time-interpolated Best Track fixes is established. For each possible storm center location on the search grid, wind speeds are transformed to Lagrangian coordinates (storm-centric) and a parametric wind model is fitted to the data. RMS error values between fit and data populate the error surface that corresponds to the search grid locations. A 2D Gaussian is fit to the error surface and the minimum of this fit is the storm center location.

not an optimal situation (Lin et al. 2013). The method is also limited by the low temporal resolution of current scatterometers. Ground-based radars are used to identify the storm center when possible, but this is only applicable for storms that are at or near landfall or already over land. Aircraft reconnaissance (recon) is also a favorable method, but it is only available for storms in the Atlantic and near Hawaii or the coast of Mexico in the Pacific.

National Hurricane Center (NHC) Best Tracks are poststorm analyses of the intensity, position, and size of TCs and represent an official historical record for each storm. The reanalysis utilizes all available data for the storm, including data with high latency that was not available in near-real time for the original operational forecast. This allows for the Best Track to make better estimates of the storm parameters than does the Working Best Track. In this work, NHC Best Tracks will be used to establish a first guess for the storm center location as well as for comparison and error analysis. The uncertainty in TC center fixes depends on the intensity of the storm as well as on the available data. For example, aircraft reconnaissance greatly reduces the amount of uncertainty in a storm center fix compared with fixes based only on satellite data. High-intensity storms typically have well-defined eyes and a high degree of organization, so the Best Track uncertainty is much lower compared to weaker, less organized storms. Overall, the uncertainty in storm fixes by NHC Best Tracks can vary from $15 \mathrm{~km}$ up to $65 \mathrm{~km}$. For more information on Best Track performance, see Landsea and Franklin (2013).

The Cyclone Global Navigation Satellite System (CYGNSS) is a NASA mission that is designed to measure ocean surface wind speed through precipitation and with sufficient frequency to resolve TC genesis and rapid intensification (Ruf et al. 2016). It was successfully launched in December 2016 into a low-Earth orbit with a $35^{\circ}$ inclination. CYGNSS makes measurements at $25-\mathrm{km}$ horizontal resolution up to $39^{\circ}$ latitude with median and mean revisit times of 3 and $7 \mathrm{~h}$, respectively. The level 2 wind speed data from this satellite system are used here to estimate the TC storm center. The data are divided into two regimes-there are two geophysical model functions (GMFs) that map the scattering cross section to wind speed. One is a fully developed seas GMF that is appropriate at wind speeds below $20 \mathrm{~m} \mathrm{~s}^{-1}$, and the other is a young seas/limited fetch GMF that is appropriate for TCs when the wind

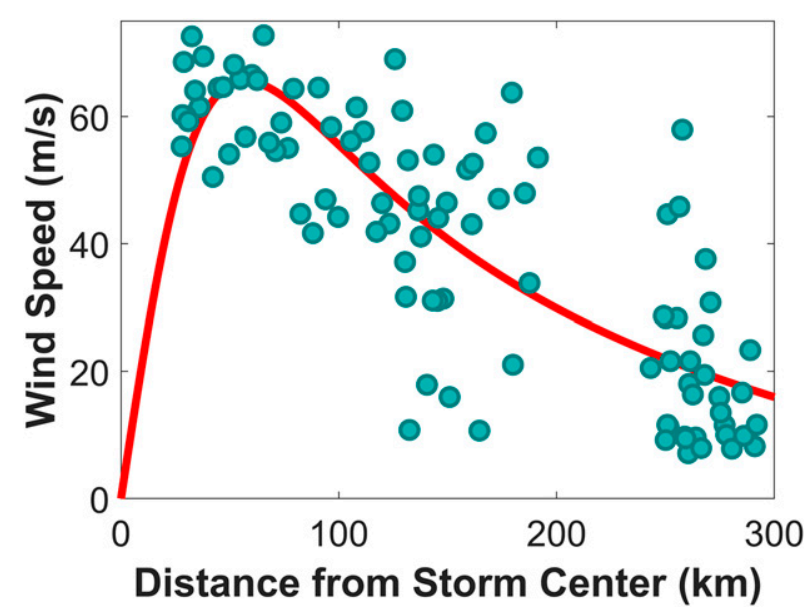

FIG. 2. Arbitrary example of parametric wind model fit to CYGNSS wind speed data. Each data point is a CYGNSS wind estimate. The red curve is the best fit to the data using the model in Morris and Ruf (2017). Note the CYGNSS winds appear noisier than reality because of the assumption of azimuthal symmetry. The error that would populate the error surface is the sum of the RMS difference between each data point and the best-fit curve (normalized by the maximum wind speed of the fit). 
speed and direction are changing significantly in intensity and direction $\left(>30 \mathrm{~m} \mathrm{~s}^{-1}\right)$. Between 20 and $30 \mathrm{~m} \mathrm{~s}^{-1}$ either GMF may be appropriate. This ambiguity is a current focus of investigation. Because this work is focused on winds near the core of TCs, the version 2.1 young seas/limited fetch winds are used here. Also note that CYGNSS is not yet properly calibrated for the Block IIF GPS satellites because of uncertainties in its antenna patterns and power fluctuations, so those data are not used.

ARCHER is an automated algorithm that fits spiral and ring shapes to satellite imagery, generating a score for each center guess over a gridded search area (Wimmers and Velden 2010, 2016). The score is reduced by a distance penalty, which is increasingly scaled by its distance from the first guess. The highest score, which may come from either the spiral or the ring, determines the center location. ARCHER storm center estimates will be compared to the NHC and CYGNSS center fixes. Note that ARCHER also relies on good organization of the storm and on the presence of an eyewall in the satellite imagery. This is not always the case for weaker storms or storms going through an eyewall replacement cycle.

\section{Algorithm description}

Storm center position is estimated by fitting a parametric wind model to CYGNSS surface wind speed data. The chosen storm center minimizes the residual error in the fit to the data. In most cases, the residual error surface as a function of possible center location has a clear minimum. The parametric wind model used follows the approach described in Morris and Ruf (2017). The model assumes a radially dependent wind field with azimuthal symmetry. Wind speed as a function of radius is described by

$$
V(r)=\frac{2 r\left(R_{m} V_{m}+0.5 f R_{m}^{2}\right)}{R_{m}^{2}+a r^{b}}-\frac{f r}{2},
$$

where $r$ is the distance from the storm center, $f$ is the Coriolis factor, $R_{m}$ is the radius of maximum winds, and $V_{m}$ is the maximum wind speed. The exponent $b$ controls the rate at which the winds radially decay away from the inner core. The factor a can be solved from the other factors by requiring that the maximum value of $V(r)$ equal $V_{m}$. So, this is effectively a threeparameter model, with parameters $b, R_{m}$, and $V_{m}$. Note that $V_{m}$ and $R_{m}$ can deviate slightly from the maximum wind speed and radius of maximum winds as $b$ varies. This wind model was chosen because the $a$ and $b$ parameters allow for a better fit to wind speed

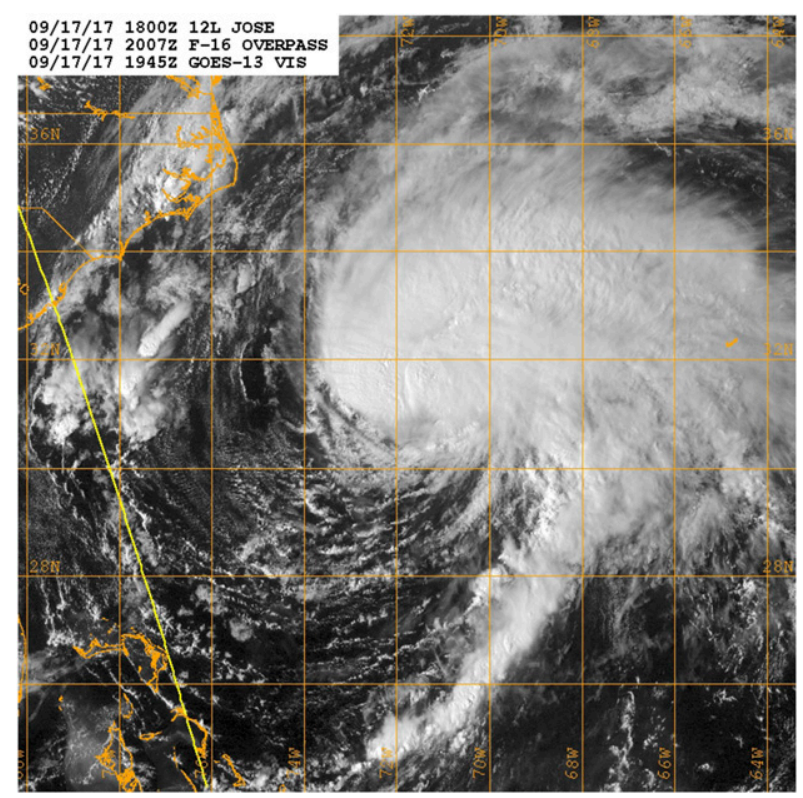

FIG. 3. Satellite imagery of Hurricane Jose at 2015 UTC 17 Sep 2017 (category 1 storm) shows disorganization and no visible eye. Since the most accurate eye-finding algorithms cannot make a high-accuracy fix, this is an opportunity for MTrack to improve upon performance.

data away from the center of the storm (Morris and Ruf 2017). The model is fit to the data using least squares minimization over the three-dimensional parameter space. The quality of fit is determined as the root-mean-square (RMS) difference between the model and observations.

A flowchart of the CYGNSS storm center fix algorithm is shown in Fig. 1. Start and end times are first selected for the population of CYGNSS data to be used. A 3-h interval is used here, although longer intervals may be worth considering. A first guess at the storm center fix is given by linear interpolation of the two NHC Best Track fixes before and after the 3-h time interval to the location at the center of the interval. Note that, while the storm is not stationary, its location (singular) refers to the storm center location at the center of the time interval. Also note that the ARCHER storm centers are more frequent than those by Best Track, so using ARCHER as a first guess would result in less interpolation error. However, it will be shown in the performance analysis section (section 4) that the MTrack algorithm is relatively insensitive to the first guess, so using ARCHER as a storm center would not have much impact on the results. Not using ARCHER also allows for unbiased comparison with ARCHER fixes.

Storm-centric regridding (transformation from Eulerian to Lagrangian coordinates) is performed on the CYGNSS 

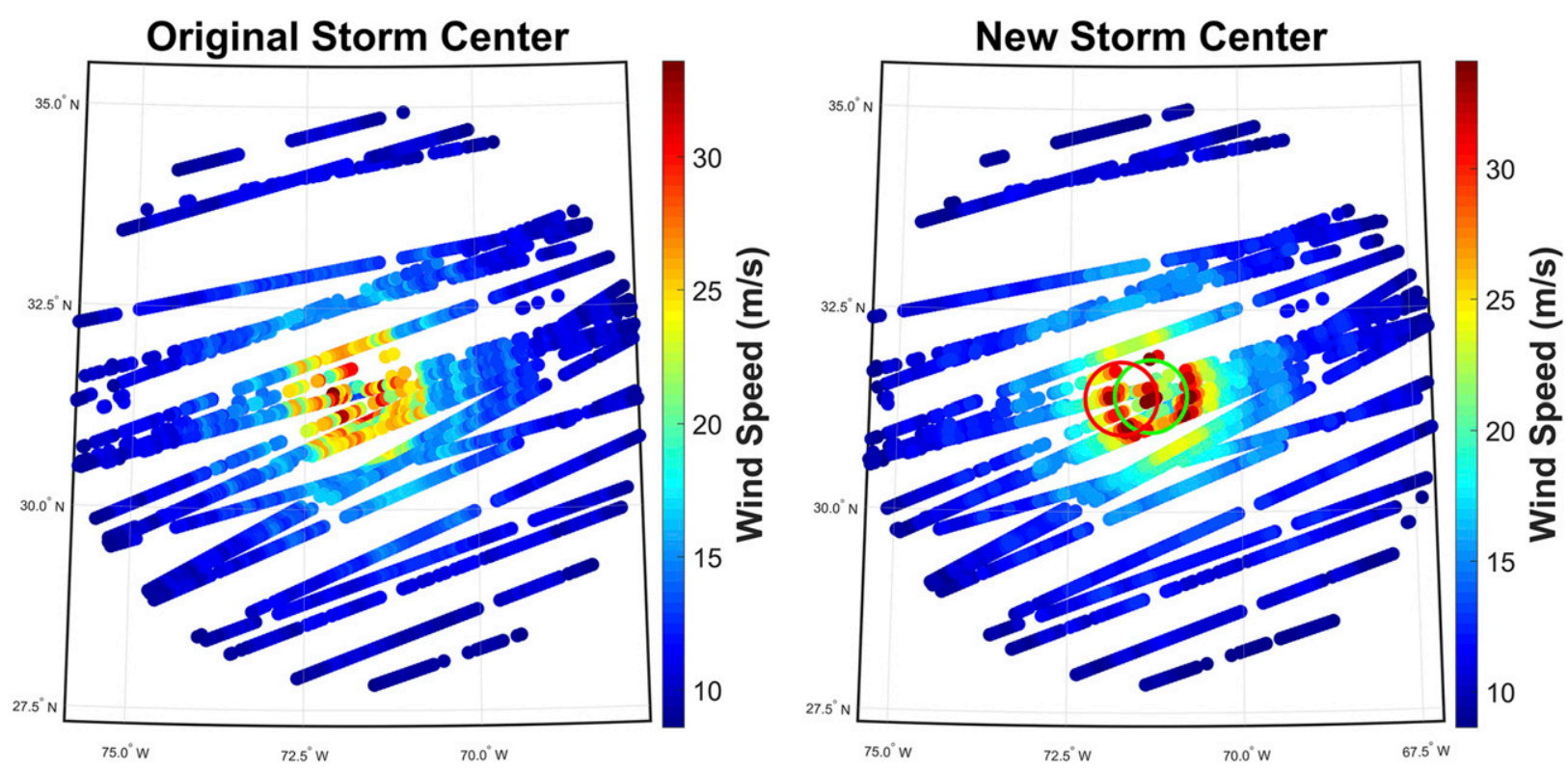

FIG. 4. CYGNSS surface wind speeds over a $3 \mathrm{~h}$ period in storm-centric coordinates (Hurricane Jose at 2015 UTC 17 Sep 2017). (left) Wind field in storm-centric coordinates relative to the original storm center location (given by interpolation from Best Track fixes). The inner core has fairly uniform wind speeds over more than a $150-\mathrm{km}$ extent. This wind structure is highly atypical and suggests that the interpolated NHC storm center fix may be incorrect. (right) Wind field in storm-centric coordinates relative to the new MTrack storm center location. The highest winds are now concentrated around the center and the winds decay radially as expected. The radius of maximum winds in this image is about $80 \mathrm{~km}$, which is close to the NHC Best Track estimate of $90 \mathrm{~km}$. Within $R_{\max }$, there are lower wind speeds, which is also characteristic of a TC. This is a significant improvement over the original wind field shown in the left panel and is strong evidence in support of the improvement in accuracy of the new storm center location.

data prior to fitting to the parametric model. For each sample, its actual location is compared to the storm center location at the sample time, assuming the storm moves in a straight-line trajectory at constant velocity between the two closest Best Track fixes. The position of the sample relative to the storm center is its stormcentric location. An example of the parametric fit to wind speed data is shown in Fig. 2. The RMS difference between the parametric model and the data constitutes one point on the residual error surface, located at the storm center assumed by the parametric model. This process is repeated over a two-dimensional grid of possible storm center locations to generate the residual error surface. Note that all CYGNSS data within $400 \mathrm{~km}$ of the assumed storm center are used, with the result that some new samples may be added and others removed from the sample population for each iteration. This process is repeated until the error surface is fully populated. The storm center location is found by fitting a 2D Gaussian function to the error surface. (Simple, direct selection of the minimum value in the error surface is not done to limit the impact of measurement noise and the presence of outliers.) Points in the error surface for which the corresponding parametric fit $R_{\max }$ is greater than $100 \mathrm{~km}$ are considered nonphysical and are not used in the Gaussian fit because TCs typically have $R_{\max }$ well below $100 \mathrm{~km}$ (Lajoie and Walsh 2008). The storm center location is given by the minimum of the $2 \mathrm{D}$ Gaussian fit to the error surface.

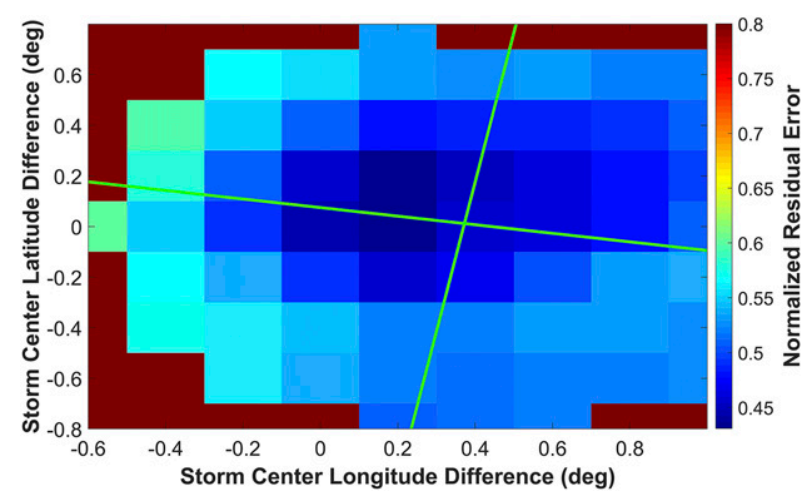

FIG. 5. A typical error surface is shown for the case of Hurricane Jose at 2015 UTC 17 Sep 2017. The point $0^{\circ}, 0^{\circ}$ is the firstguess location given by interpolation of Best Track fixes. Each grid point is the RMS difference between the parametric wind model fit and the CYGNSS wind speed data normalized by the maximum wind speed of the fit $\left(V_{\max }=27 \mathrm{~m} \mathrm{~s}^{-1}\right)$. The red points are masked out because they are unphysical $\left(R_{\max }\right.$ of fit greater than $100 \mathrm{~km})$. The intersecting lines are the 2D Gaussian fit to the error surface. The minimum of the 2D Gaussian, the storm center location, is the intersection of the green lines. 


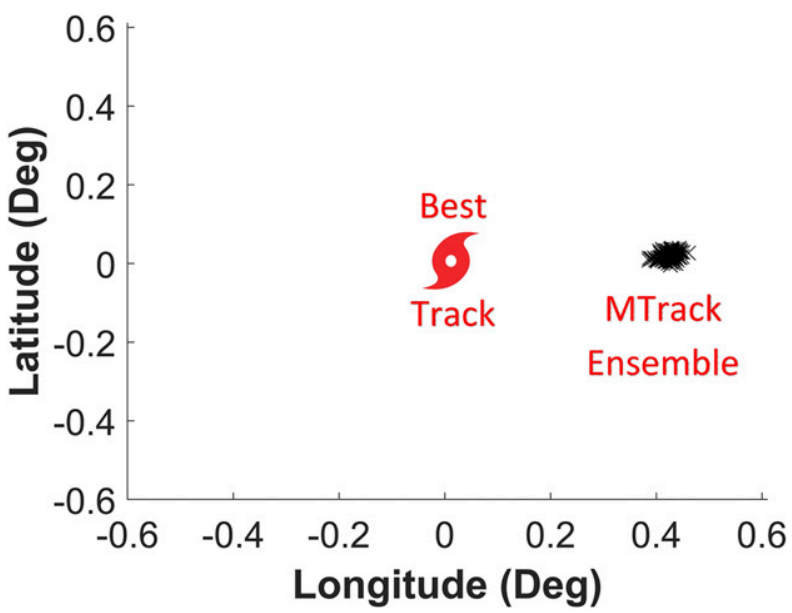

FIG. 6. Ensemble of storm centers for Hurricane Jose at 201517 Sep 2017: 100 trials were run with perturbed NHC Best Track fixes used as input (radially perturbed by a Gaussian with a standard deviation of $10 \mathrm{~km}$ ). The first guess, which is at $0^{\circ}, 0^{\circ}$, is given by linear interpolation of the NHC Best Track fixes. Each black cross is the refined storm center location for each of the 100 trials. The lack of scatter in the results of the 100 trials demonstrates how MTrack is insensitive to error in Best Track input. This is important for producing an independent estimate of the storm center location. Also note that the uncertainty in storm center was reduced from $10 \mathrm{~km}$ for the Best Track input to $1.73 \mathrm{~km}$ for the MTrack output.

To reduce the effects of errors in the NHC storm center fixes on this algorithm, an ensemble averaging approach is used, similar to how TC paths are forecast (Zhang and Krishnamurti 1997, 1999). The initial NHC fixes are randomly perturbed in each trial, resulting in an ensemble of storm center estimates. The radial perturbation about the NHC fixes is a zero mean Gaussian with a specified standard deviation, and the azimuthal angle is uniformly distributed between $0^{\circ}$ and $360^{\circ}$. The standard deviation of the perturbing Gaussian varies between 10 and $70 \mathrm{~km}$ depending on storm organization and the types of observations available (Landsea and Franklin 2013). Each NHC storm center is perturbed independently. The final storm center estimate is the average over the ensemble. We refer to this as the MTrack estimate.

\section{Examples of MTrack storm center fixes by CYGNSS}

Two examples of the MTrack storm center fix are given to illustrate its application and demonstrate some characteristics of its performance. The first, with Atlantic Hurricane Jose on 17 September 2017, is for a weakly organized storm where the fix changes significantly from the interpolated NHC location. The second example, for Pacific Hurricane Sergio on 7 October

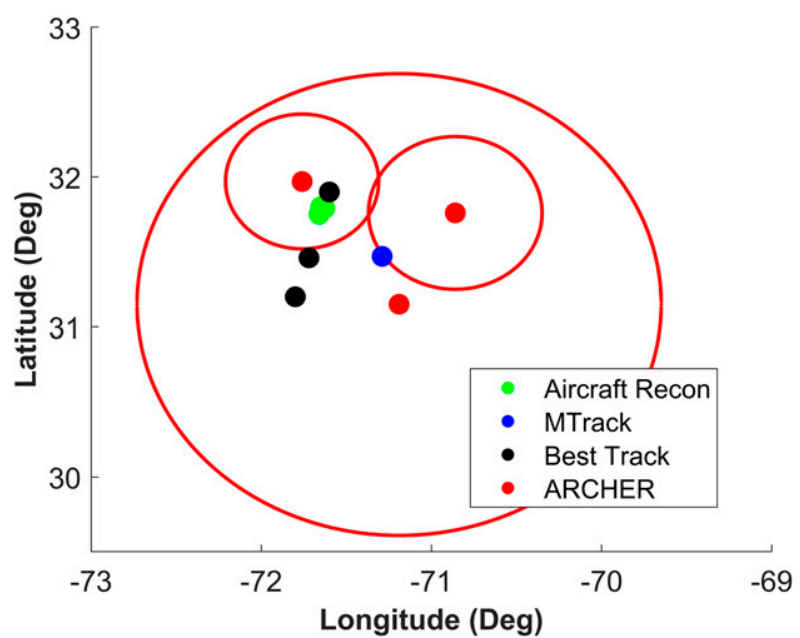

FIG. 7. Comparison of storm center locations from MTrack, NHC Best Track, ARCHER, and aircraft reconnaissance for Hurricane Jose on 17 Sep 2017. The storm is moving to the northeast. The three Best Track fixes are at 1800 and 0000 UTC, with the intermediate point as the linearly interpolated center (first guess for the MTrack algorithm). The MTrack fix is at 2015 UTC. The ARCHER fixes are at 1745, 2008, and 2258 UTC. The aircraft recon fixes are at 2207, 2313, and 2329 UTC. The circles around the ARCHER fixes are the $95 \%$ confidence radii.

2017, is a case where the MTrack fix is consistent with NHC.

Hurricane Jose is considered for the 3-h interval centered on 2015 UTC 17 September 2017 (category 1 storm). This is a high-latitude case $\left(\sim 32^{\circ} \mathrm{N}\right)$ for which CYGNSS has a large number of samples. Satellite imagery at this time (Fig. 3) shows weak organization and lack of a clear eye, both of which can limit the accuracy of a conventional storm center fix. This is an opportunity for CYGNSS to improve upon the storm center location. Figure 4a shows the wind field measured by CYGNSS in storm-centric coordinates, assuming the interpolated NHC storm center fix is correct, and using a $10-\mathrm{km}$ radial smoothing to reduce measurement noise. The inner core has fairly uniform wind speeds over more than a $150-\mathrm{km}$ extent. This wind structure is highly atypical and suggests that the interpolated NHC storm center fix may be incorrect.

Figure 5 shows the residual error surface for a range of possible storm center locations, together with the 2D Gaussian fit to the surface. Each point in the grid is the RMS difference between the parametric wind fit and the CYGNSS wind speed data, which is then normalized by the maximum wind speed. The crosshairs in the figure indicate the location of the minimum in the Gaussian fit, which would be the estimated storm center location without ensemble averaging. The red grid points indicate cases for which $R_{\max }>100 \mathrm{~km}$ in the 


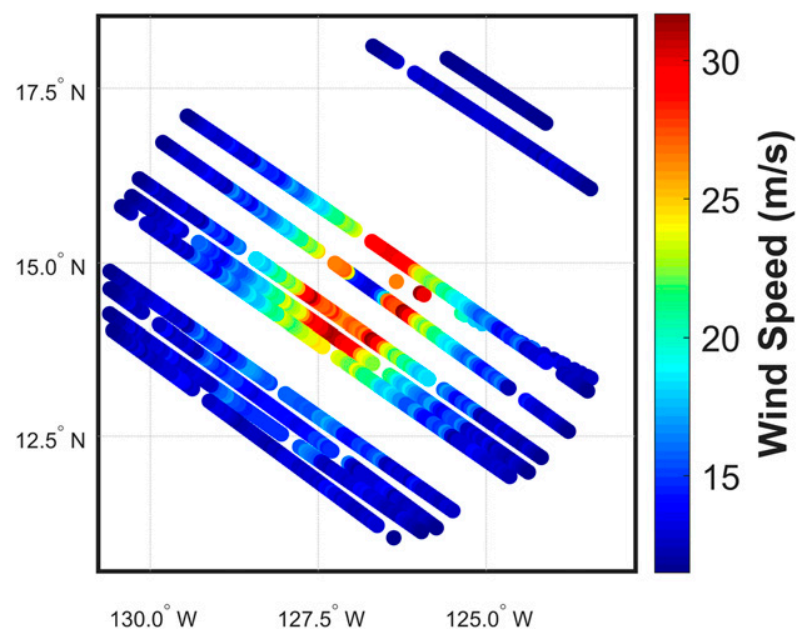

FIG. 8. CYGNSS surface wind speeds over a 3-h period in stormcentric coordinates relative to the original storm center location (Hurricane Sergio at 1330 UTC 7 Oct 2018). In this case, the original storm center produces a wind profile that looks characteristic of a typical tropical cyclone, and the original storm center within the eye is resolved by CYGNSS. Because the first guess is reasonable, the MTrack algorithm is expected to produce a storm center location close to the first guess.

best-fit parametric model. Those points are not considered by the Gaussian fit. Note that the center location is about $0.2^{\circ}$ away from the grid point with minimum RMS error.
Figure 6 shows the results after ensemble averaging. 100 trials were performed with NHC storm center locations perturbed by a zero mean Gaussian with a standard deviation of $10 \mathrm{~km}$, and the resulting storm center for each trial is indicated by a black cross. The green cross marks the average over all trials (i.e., the MTrack fix). The red dot is the initial storm center determined by linear interpolation of NHC storm centers. Note that the individual storm center fixes are fairly insensitive to perturbations in the NHC center, as indicated by their tight clustering. This suggests that the ensemble-averaged storm center should not be affected much by Best Track errors.

A new storm-centric, radially smoothed wind field is constructed using the MTrack storm center location and is shown in Fig. 4b. The wind field now has its highest winds concentrated around the center and the winds decay radially as expected. The radius of maximum winds in this image is about $80 \mathrm{~km}$, which is close to the operational estimate in the ATCF file: $90 \mathrm{~km}$. Within $R_{\max }$, there are lower wind speeds, which is also characteristic of a TC. This is a significant improvement over the original wind field shown in Fig. 4a and is strong evidence in support of the improvement in accuracy of the new storm center location.

The new CYGNSS center fix is independently validated by aircraft recon and ARCHER. Figure 7 plots

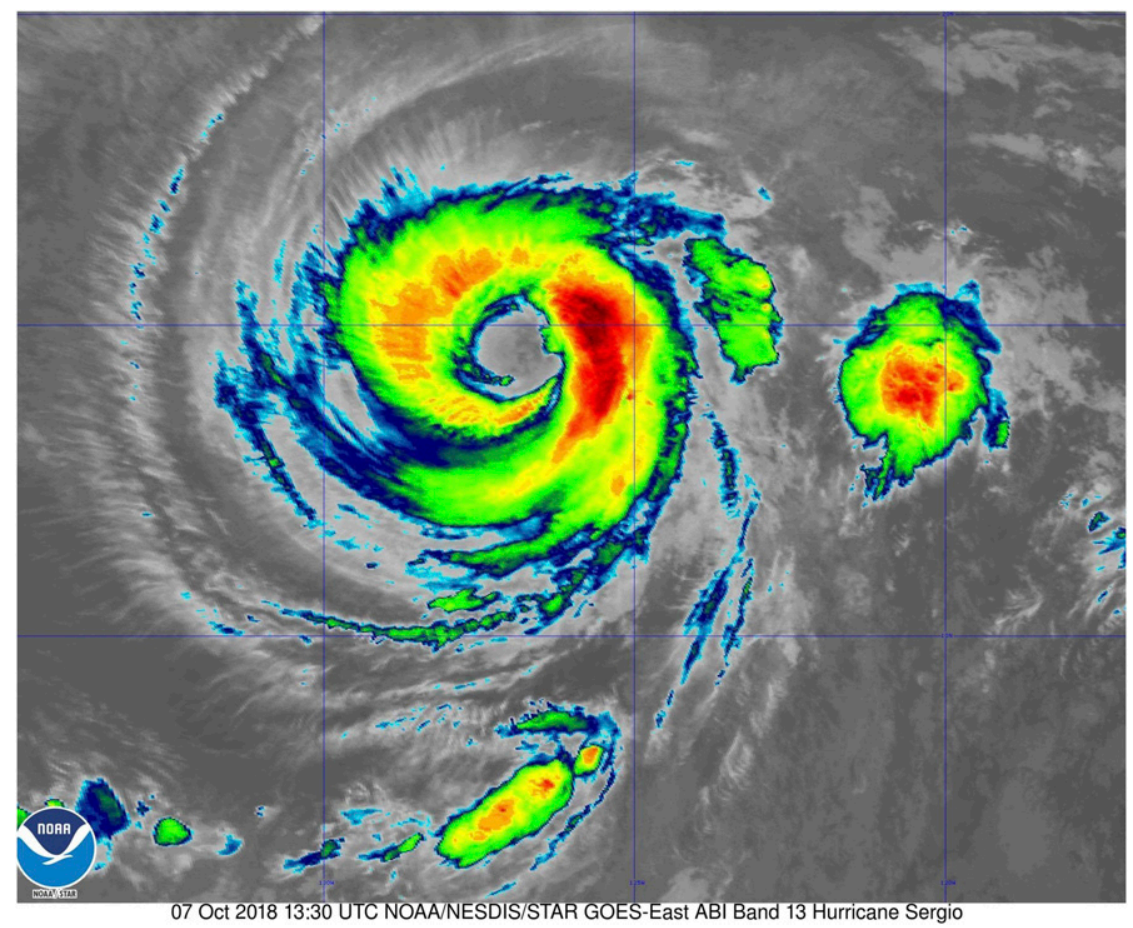

FIG. 9. Satellite imagery of Hurricane Sergio at 1330 UTC 7 Oct 2018 shows a highly organized storm with a very large eye. This explains why CYGNSS, which has a resolution of about $25 \mathrm{~km}$, can resolve the eye in this case (Fig. 8). 


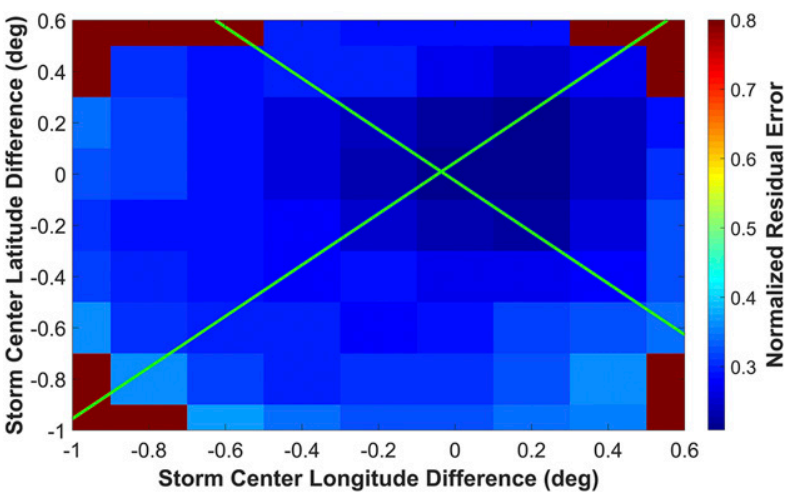

FIG. 10. A typical error surface is shown for the case of Hurricane Sergio at 1330 UTC 7 Oct 2018 . The point $0^{\circ}, 0^{\circ}$ is the first guess location given by interpolation of Best Track fixes. Each grid point is the RMS difference between the parametric wind model fit and the CYGNSS wind speed data normalized by the maximum wind speed of the fit $\left(V_{\max }=29 \mathrm{~m} \mathrm{~s}^{-1}\right)$. The red points are masked out because they are unphysical $\left(R_{\max }\right.$ of fit greater than $\left.100 \mathrm{~km}\right)$. The intersecting lines are the 2D Gaussian fit to the error surface. The minimum of the 2D Gaussian, the storm center location, is the intersection of the green lines. As expected, the MTrack fix is very close to the first guess.

the new MTrack storm center together with the ARCHER, aircraft recon, and NHC Best Track fixes. The aircraft recon and Best Track centers agree very well (Best Track probably used the aircraft recon fixes as input after all). Two ARCHER fixes are over half a degree to the east of the NHC Best Track fixes, and one Best Track fix even lies outside of the $95 \%$ confidence radius of the ARCHER fix. The CYGNSS storm center location is not quite as far east as ARCHER. It represents a compromise between all available fixes since it is between Best Track and ARCHER, while still inside ARCHER's 95\% confidence radius. A third estimate of the storm center can help disambiguate the fix in cases where ARCHER and Best Track do not agree, or in cases like this it can indicate that the answer is in the middle.

The second example considers category 2 Hurricane Sergio in the Pacific using $3 \mathrm{~h}$ of CYGNSS measurements centered on 1330 UTC 7 October 2017. The latitude of this storm is about $15^{\circ} \mathrm{N}$ so there is a lower spatial density of CYGNSS samples. Figure 8 shows the initial wind field (assuming the interpolated NHC fix is correct) with highest winds near the eyewall and radial decay away from the center. CYGNSS resolves the eye in this case because Sergio had an unusually large eye, as seen in the satellite imagery in Fig. 9. Since the initial NHC fix appears to be quite good, this is a good case to test whether the MTrack algorithm estimates a similar storm center location.

The residual error surface and its 2D Gaussian fit are shown in Fig. 10. A distinct minimum in the error

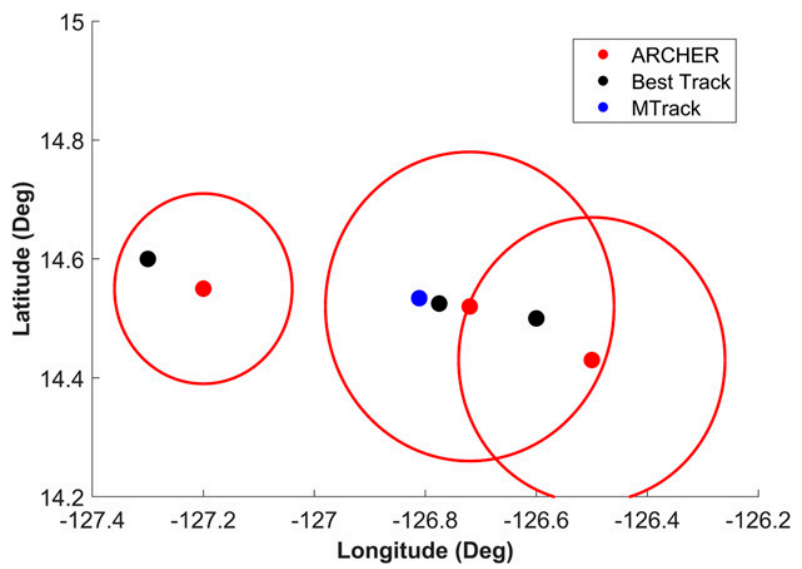

FIG. 11. Comparison of storm center locations from MTrack, NHC Best Track, and ARCHER for Hurricane Sergio on 7 Oct 2018. The storm is moving to the northwest. The black dots are Best Track fixes at 1200, 1330, and 1800 UTC with the intermediate point obtained from the other two by linear interpolation. The blue dot is the MTrack storm center at 1330 UTC. The red dots are ARCHER fixes at 1200, 1411, and 1800 UTC. The red circles around the ARCHER fixes are the $95 \%$ confidence radii. All three storm center databases agree for these times, which is expected for a storm with such a clear eye.

surface is evident where the storm center is located. The MTrack center has moved less than $4 \mathrm{~km}$ from the NHC fix. Figure 11 combines the CYGNSS, NHC, and ARCHER storm center locations (aircraft recon data are not available for this time). Because the eye is so clear, there is little uncertainty in the ARCHER fix. Between 1200 and 1800 UTC 7 October, both the Best Track centers and the CYGNSS MTrack center fall within the $95 \%$ confidence radii of ARCHER.

\section{Performance analysis}

A single CYGNSS storm center estimate is made as a refinement to the linear interpolation of the pair of NHC Best Track storm center fixes, one before and one after the 3-h window of CYGNSS wind speed measurements used by the estimator. An ensemble of storm center estimates is produced by randomly perturbing those two NHC fixes by a Gaussian with a specified standard deviation. The average across the ensemble of CYGNSS estimates is the MTrack fix, which we consider our best estimate of the storm center location. The performance of the MTrack fix is characterized in two ways. Its uncertainty is defined as the standard deviation of the ensemble fixes. This interpretation is borrowed from the concept of "cone of uncertainty" in ensemble forecasting of TC track. The uncertainty will depend on the standard deviation of the Gaussian perturbations made to the NHC fixes 


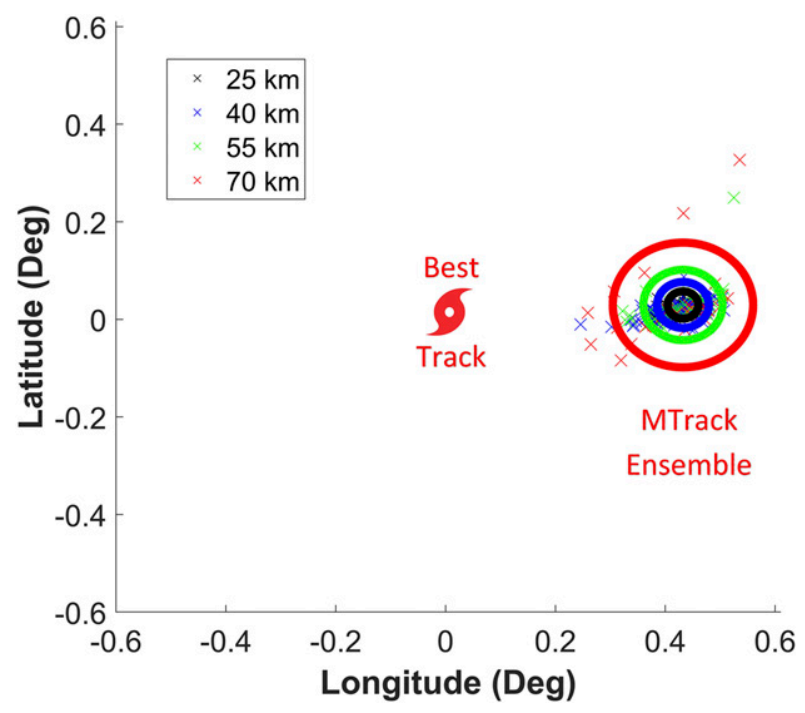

FIG. 12. Monte Carlo trial storm centers for Hurricane Jose at 2015 UTC 17 Sep 2017: 100 trials were run with perturbed NHC Best Track fixes as input (radially perturbed by Gaussians with standard deviations of $25,40,55$, and $70 \mathrm{~km})$. The first guess location is at $0^{\circ}, 0^{\circ}$. Each cross is a storm center location for a trial that had a standard deviation as given by the color of the cross. The circles around the ensemble indicate the standard deviation from the mean center. As Best Track uncertainty increases, the standard deviation of the fix increases, but they remain tightly clustered with respect to the first guess and the search grid. In all cases uncertainty is significantly reduced by MTrack. Also shown is the eccentricity of the trial storm centers. There is more uncertainty in the cross-path direction, which is characteristic of the MTrack algorithm.

when generating the ensemble population. A second MTrack performance characteristic is the ratio between the assumed error in the NHC fix (the standard deviation of the perturbing Gaussian) and the resulting uncertainty in the MTrack fix. This ratio, henceforth called the uncertainty reduction factor, represents the reduction in storm center fix uncertainty that results with the MTrack algorithm. This is a unitless value.

An example of MTrack performance uses CYGNSS wind speed measurements of Hurricane Jose made over a 3-h interval centered on 2015 UTC 17 September 2017. The corresponding MTrack fix was computed in the previous section. A similar computation is considered here with larger perturbations in the NHC Best Track fixes. The standard deviation of the perturbations is increased from 10 to $25,40,55$, and $70 \mathrm{~km}$. These values correspond to the levels of uncertainty in the NHC fix associated with varying levels of storm organization and with the different types of observations used to produce an NHC fix. An ensemble of 100 trials was assembled for each value of standard deviation. The resulting distributions of individual CYGNSS storm center fixes are shown graphically in Fig. 12. The point
TABLE 1. For Hurricane Jose at 2015 UTC 17 Sep 2017, the behavior of the MTrack algorithm throughout the entire range of NHC Best Track storm center errors [or perturbation standard deviations (Std devs)]. As the perturbation grows larger, the uncertainty of the MTrack fix also becomes larger, but the uncertainty reduction factor is approximately constant over the entire range. This suggests that the MTrack algorithm produces a consistent improvement in confidence even at the highest values of NHC uncertainty.

\begin{tabular}{ccc}
\hline \hline $\begin{array}{c}\text { Perturbation std } \\
\text { dev }(\mathrm{km})\end{array}$ & $\begin{array}{c}\text { Uncertainty of } \\
\text { fix }(\mathrm{km})\end{array}$ & $\begin{array}{c}\text { Uncertainty reduction } \\
\text { factor }\end{array}$ \\
\hline 10 & 1.73 & 5.78 \\
25 & 3.10 & 8.06 \\
40 & 5.49 & 7.28 \\
55 & 6.77 & 8.12 \\
70 & 7.45 & 9.39 \\
\hline
\end{tabular}

$0^{\circ}, 0^{\circ}$ in the figure is the storm center estimated by linear interpolation of the unperturbed NHC fixes without consideration of the CYGNSS measurements. The crosses are the storm center calculations using CYGNSS measurements for each trial in the ensemble. The color of the cross denotes the uncertainty of the Best Track fix that was used as input for that trial (see legend of Fig. 12). The MTrack uncertainty and uncertainty reduction factor are computed from these trial populations. The results are tallied in Table 1 . The MTrack uncertainty increases monotonically with that of the NHC fix and the ratio between the two (the uncertainty reduction factor) is relatively constant once the NHC uncertainty is $25 \mathrm{~km}$ or greater. This suggests that the MTrack algorithm produces a consistent improvement in confidence even at the highest values of NHC uncertainty.

The above performance assessment was repeated for a number of other storm cases: Hurricane Sergio at 1200 UTC 7 October 2018, Hurricane Maria at 2300 UTC 17 September 2017, Hurricane Jose at 1830 UTC 16 September 2017, and Hurricane Florence at 1730 UTC 12 September 2018. These cases were chosen because they are well sampled by CYGNSS. The MTrack uncertainty and uncertainty reduction factor in each case are listed in Table 2 for an NHC uncertainty of $40 \mathrm{~km}$ [a typical uncertainty value according to Landsea and Franklin (2013)]. This NHC storm center uncertainty is an average value, which is meant to give a sense of typical algorithm performance. The resulting MTrack uncertainties range from 5.49 to $8.97 \mathrm{~km}$ and the average uncertainty reduction factor across all storm cases is 5.56. The variation in MTrack uncertainty is likely due to a number of factors, including differences in spatial sampling density and distribution, degree of storm organization, and storm center movement during the time interval of data collection. 
TABLE 2. The primary performance metrics, uncertainty of fix and uncertainty reduction factor, for five different cases. The category of the TC, based on the Saffir-Simpson scale, is also included. For each case, 100 trials were performed with randomly perturbed NHC Best Track storm centers as input. The standard deviation of these 100 trials is the uncertainty of the fix. The Best Track input uncertainty divided by the MTrack uncertainty is the uncertainty reduction factor. The uncertainty reduction factor is much greater than 1 in all cases, meaning that MTrack always reduces the uncertainty compared to Best Track.

\begin{tabular}{lccc}
\hline \hline \multicolumn{1}{c}{ Case } & MTrack uncertainty $(\mathrm{km})$ & Uncertainty reduction factor & Hurricane category \\
\hline Hurricane Jose at 2015 UTC 17 Sep 2017 & 5.49 & 7.28 & 1 \\
Hurricane Maria at 0030 UTC 18 Sep 2017 & 8.97 & 4.46 & 1 \\
Hurricane Sergio at 1330 UTC 7 Oct 2018 & 7.61 & 5.25 & 2 \\
Hurricane Jose at 2000 UTC 16 Sep 2017 & 8.26 & 4.84 & 1 \\
Hurricane Florence at 1900 UTC 12 Sep 2018 & 6.68 & 5.98 & 3 \\
\hline
\end{tabular}

The MTrack uncertainty has thus far been considered as a scalar, averaged over all directions without regard for possible azimuthal dependence. As Fig. 12 suggests, however, there is an asymmetric spread in the individual storm center fixes about the ensemble average. The asymmetry manifests itself as a larger uncertainty in the component of the storm center fix that is perpendicular to the direction of storm center motion (henceforth referred to as cross path). The component of uncertainty parallel to the direction of storm center motion, or along-path, is typically 1-2 times smaller. Table 3 summarizes the cross-pathalong-path asymmetry in uncertainty for several cases considered. In all cases, the cross-path uncertainty is greater than the along-path uncertainty, with the ratio varying between 1.3 and 2.2. The asymmetry appears to stem from a similar asymmetry in the error surface and is directly related to the wind field measured by CYGNSS. The underlying cause or causes of the asymmetry requires more analysis. If confirmed, it implies that MTrack has higher certainty in the cross-path direction than the along-path direction.

MTrack performance was also examined with respect to the uncertainty in the CYGNSS wind speeds themselves via another Monte Carlo simulation. For each of 100 trials, CYGNSS wind measurements were perturbed by zero mean Gaussian random noise with standard deviation given by the uncertainty value reported in the level 2 data product files. For more information on how CYGNSS uncertainties are determined, see
Gleason et al. (2019) and Ruf et al. (2019). The storm center was estimated for each trial, and the standard deviation of the fix was computed for the 100 trials. This process was done for the five cases previously described. Table 4 summarizes the results. The standard deviation ranges from 3 to $9 \mathrm{~km}$ for these cases, with an average value of $5.7 \mathrm{~km}$. This represents the uncertainty in MTrack due to CYGNSS wind speed measurement uncertainty. Figure 13 shows the wind perturbation results for the overpass of Hurricane Jose on 2015 UTC 17 September 2017, which is a typical result. The green cross is the MTrack storm center estimate (made without wind speed perturbations) and the red cross is the mean of the 100 trials with wind speed perturbations. The difference between them is $0.6 \mathrm{~km}$, suggesting that the wind speed measurement noise introduces little if any bias in the MTrack fix.

\section{Discussion}

It has been shown that errors in CYGNSS winds cause an average uncertainty of $5.7 \mathrm{~km}$ in the MTrack fix, and uncertainties in NHC Best Track fixes are reduced on average by a factor of 5.6. There are other potential sources of error that have not yet been quantified that should also be considered when estimating the overall uncertainty. The MTrack algorithm assumes an azimuthally symmetric wind field, which is never true, but especially so in weaker, more disorganized storms. It remains to be investigated how much

TABLE 3. Comparison of cross-path and along-path uncertainty. The cross-path uncertainty is greater than the along-path uncertainty in all cases. This seems to stem from a similar eccentricity in the error surface.

\begin{tabular}{lcc}
\hline \hline \multicolumn{1}{c}{ Case } & MTrack uncertainty (km) & $\begin{array}{c}\text { Ratio of cross-path uncertainty to } \\
\text { along-path uncertainty }\end{array}$ \\
\hline Hurricane Jose at 2015 UTC 17 Sep 2017 & 5.49 & 1.29 \\
Hurricane Maria at 0030 UTC 18 Sep 2017 & 8.97 & 1.49 \\
Hurricane Sergio at 1330 UTC 7 Oct 2018 & 7.61 & 1.54 \\
Hurricane Jose at 2000 UTC 16 Sep 2017 & 8.26 & 2.16 \\
Hurricane Jose at 0500 UTC 9 Sep 2017 & 22.4 & 1.35 \\
Typhoon Shanshan at 1915 UTC 7 Aug 2018 & 18 & 1.72 \\
\hline
\end{tabular}


TABLE 4. Resulting uncertainties in fix due to CYGNSS wind speed errors for many cases. For each case, CYGNSS wind speeds were perturbed by a Gaussian with a standard deviation equal to the uncertainty in the measurement of the wind. By running many Monte Carlo trials, the uncertainty in storm center location that is generated by wind speed error was calculated.

\begin{tabular}{lc}
\hline \hline \multicolumn{1}{c}{ Case } & Uncertainty $(\mathrm{km})$ \\
\hline Hurricane Maria at 0045 UTC 20 Sep 2017 & 3.71 \\
Hurricane Jose at 2015 UTC 17 Sep 2017 & 4.94 \\
Hurricane Maria at 0030 UTC 18 Sep 2017 & 5.11 \\
Hurricane Sergio at 1330 UTC 7 Oct 2018 & 4.64 \\
Hurricane Jose at 2000 UTC 16 Sep 2017 & 7.33 \\
Hurricane Florence at 1900 UTC 12 Sep 2018 & 9.01 \\
\hline
\end{tabular}

error is contributed by the lack of symmetry and how much this error increases as the storm becomes increasingly disorganized. MTrack performance was demonstrated for category 1-3 hurricanes in this paper, but tropical storm performance remains to be characterized in a future comprehensive error analysis. Other errors may arise from linear interpolation of the Best Track storm centers. Linear interpolation restricts storm motion to a straight line with constant speed over $6 \mathrm{~h}$. Judging by the performance of storm centric coordinates in the smoothed wind field and in the storm center fixes, this seems to be a good assumption most of the time, but further analysis is needed to evaluate how often this assumption is valid and, when it is not, how much the curvature in the path or acceleration in the storm motion can affect the fix.

It has been shown that the MTrack uncertainty reduction factor is significantly greater than 1 in all cases. This implies that the MTrack algorithm produces a storm center location at an intermediate time between NHC fixes with improved precision. Note that MTrack uncertainty has been defined as the standard deviation of the ensemble of fixes and is not necessarily a measure of the absolute accuracy of the MTrack fix. This would be a more difficult metric to develop, requiring knowledge of the true storm center location, or assuming that Best Track centers are "truth."

The MTrack storm center locations will be able to fill in temporal gaps in other datasets, provide additional estimates for comparison, and provide an improved fix when other methods have difficulty. It is unlikely that the MTrack fix will be able to improve upon fixes made from satellite imagery of a clear eye or from aircraft reconnaissance. However, in the case of aircraft reconnaissance, MTrack can be used to expand the temporal resolution of the fix outside of the aircraft overpass times. There is a limited number of aircraft, and they are especially limited in their temporal sampling when
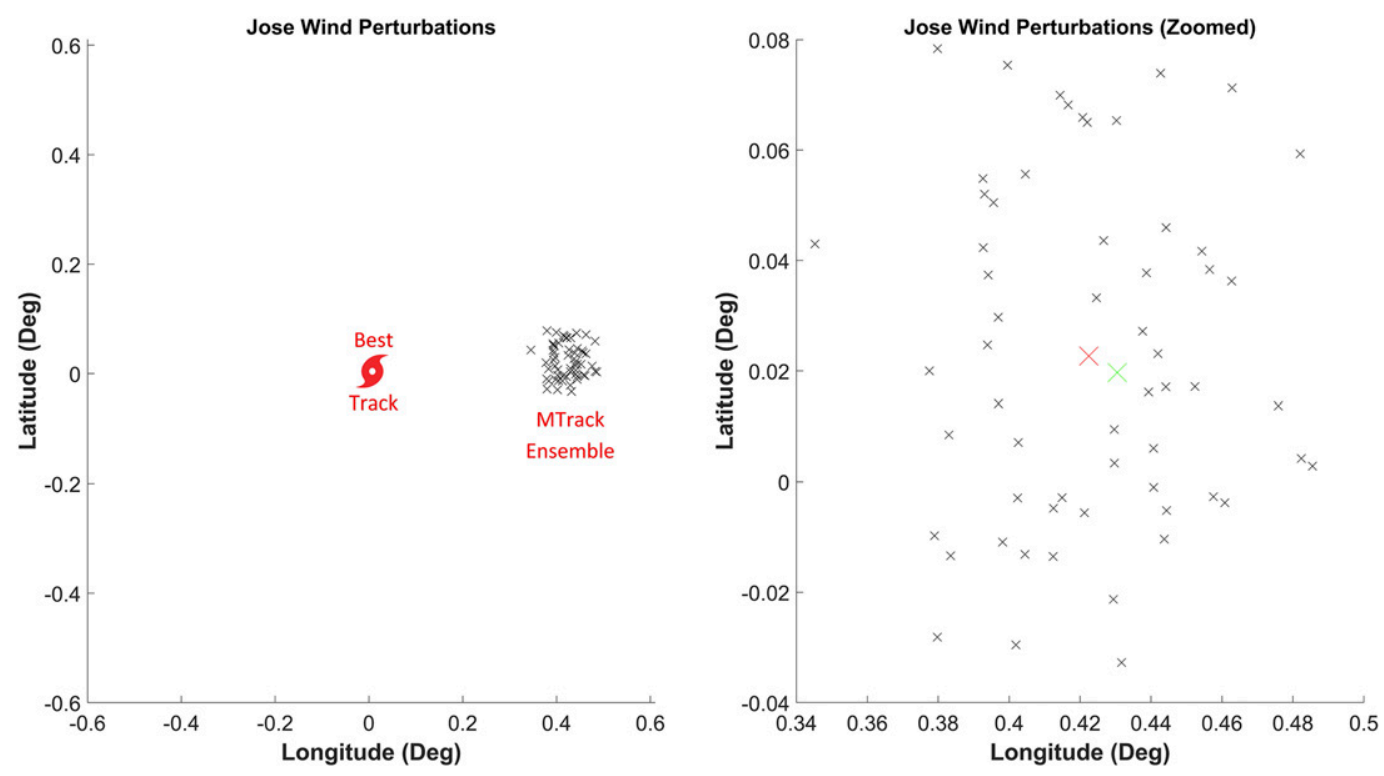

FIG. 13. The first guess (interpolated from Best Track) is located at $0^{\circ}, 0^{\circ}$. The cluster of black crosses represents the fixes of each perturbation trial. For each trial, CYGNSS surface wind speeds were perturbed by a Gaussian of standard deviation equal to the uncertainty in the CYGNSS data. (left)The algorithm produces storm center locations with high certainty compared to the size of the search grid, even in the presence of error in the input wind speeds. (right) Zoom in on the ensemble. The red cross is the average of all trials while the green cross is the MTrack storm center, our best estimate of the storm center location. Although the error in wind speeds causes an uncertainty of $4.94 \mathrm{~km}$, the mean of all wind speed perturbation trials is only $0.57 \mathrm{~km}$ from the MTrack center. This algorithm is very robust to input error. 
a storm is far from land. In the case of satellite imagery of a clear eye, geostationary satellites downlink multiple images per hour, so the temporal resolution is excellent. An MTrack fix would serve as a second estimate of the storm center location. However, recall that eyes are only visible in $12.6 \%$ of images of TCs. In the more typical case of a storm with a shrouded eye or disorganization, scatterometers are often used to determine the fix. Fixes based on satellite scatterometers are generally less accurate than methods that fit to the eyewall or rainbands, and they also suffer from relatively poor temporal resolution. The MTrack fix will improve upon the temporal sampling because of CYGNSS's short revisit time. MTrack also has the potential to improve upon the fixes from scatterometers since they suffer from performance degradation in heavy precipitation while CYGNSS is unaffected. The performance of MTrack in situations with storm disorganization requires more investigation, but this is another potential opportunity for MTrack to improve upon the accuracy of current storm center fix techniques. It should also be noted that MTrack has the potential to be used operationally since its uncertainty reduction factor is valid even at the higher center fix uncertainty levels that are typical of near-real-time, operational fixes.

Acknowledgments. The work presented was supported in part by NASA Science Mission Directorate Contract NNL13AQ00C with the University of Michigan.

\section{REFERENCES}

Bai, Q. C., K. Wei, Z. L. Jing, Y. X. Li, H. Y. Tuo, and C. G. Liu, 2012: Tropical cyclone spiral band extraction and center locating by binary ant colony optimization. Sci. China Earth Sci., 55, 332-346, https://doi.org/10.1007/s11430-011-4334-7.

Gleason, S., C. S. Ruf, A. J. O'Brien, and D. S. McKague, 2019: The CYGNSS level 1 calibration algorithm and error analysis based on on-orbit measurements. IEEE J. Sel. Top. Appl. Earth Obs. Remote Sens., 12, 37-49, https://doi.org/10.1109/ JSTARS.2018.2832981.

Kieper, M. E., and H. Jiang, 2012: Predicting tropical cyclone rapid intensification using the $37 \mathrm{GHz}$ ring pattern identified from passive microwave measurements. Geophys. Res. Lett., 39, L13804, https://doi.org/10.1029/2012GL052115.

Knapp, K. R., M. C. Kruk, D. H. Levinson, H. J. Diamond, and C. J. Neumann, 2010: The International Best Track Archive for
Climate Stewardship (IBTrACS). Bull. Amer. Meteor. Soc., 91, 363-376, https://doi.org/10.1175/2009BAMS2755.1.

, C. S. Velden, and A. J. Wimmers, 2018: A global climatology of tropical cyclone eyes. Mon. Wea. Rev., 146, 2089-2101, https://doi.org/10.1175/MWR-D-17-0343.1.

Kruk, M. C., K. R. Knapp, and D. H. Levinson, 2010: A technique for combining global tropical cyclone best track data. J. Atmos. Oceanic Technol., 27, 680-692, https://doi.org/ 10.1175/2009JTECHA1267.1.

Lajoie, F., and K. Walsh, 2008: A technique to determine the radius of maximum wind of a tropical cyclone. Wea. Forecasting, 23, 1007-1015, https://doi.org/10.1175/2008WAF2007077.1.

Landsea, C. W., and J. L. Franklin, 2013: Atlantic hurricane database uncertainty and presentation of a new database format. Mon. Wea. Rev., 141, 3576-3592, https://doi.org/10.1175/ MWR-D-12-00254.1.

Lin, W., M. Portabella, A. Stoffelen, and A. Verhoef, 2013: On the characteristics of ASCAT wind direction ambiguities. Atmos. Meas. Tech., 6, 1053-1060, https://doi.org/10.5194/amt-6-1053-2013.

Morris, M., and C. S. Ruf, 2017: Determining tropical cyclone surface wind speed structure and intensity with the CYGNSS satellite constellation. J. Appl. Meteor. Climatol., 56, 1847-1865, https:// doi.org/10.1175/JAMC-D-16-0375.1.

Olander, T. L., and C. S. Velden, 2007: The advanced Dvorak technique: Continued development of an objective scheme to estimate tropical cyclone intensity using geostationary infrared satellite imagery. Wea. Forecasting, 22, 287-298, https:// doi.org/10.1175/WAF975.1.

Rozoff, C. M., C. S. Velden, J. Kaplan, J. P. Kossin, and A. J. Wimmers, 2015: Improvements in the probabilistic prediction of tropical cyclone rapid intensification with passive microwave observations. Wea. Forecasting, 30, 1016-1038, https:// doi.org/10.1175/WAF-D-14-00109.1.

Ruf, C. S., and Coauthors, 2016: New ocean winds satellite mission to probe hurricanes and tropical convection. Bull. Amer. Meteor. Soc., 97, 385-395, https://doi.org/10.1175/BAMS-D-14-00218.1.

- S. Gleason, and D. S. McKague, 2019: Assessment of CYGNSS wind speed retrieval uncertainty. IEEE J. Sel. Top. Appl. Earth Obs. Remote Sens., 12, 87-97, https://doi.org/ 10.1109/JSTARS.2018.2825948.

Wimmers, A. J., and C. S. Velden, 2010: Objectively determining the rotational center of tropical cyclones in passive microwave satellite imagery. J. Appl. Meteor. Climatol., 49, 2013-2034, https://doi.org/10.1175/2010JAMC2490.1.

$\longrightarrow$, and —, 2016: Advancements in objective multisatellite tropical cyclone center fixing. J. Appl. Meteor. Climatol., 55, 197-212, https://doi.org/10.1175/JAMC-D-15-0098.1.

Zhang, Z., and T. N. Krishnamurti, 1997: Ensemble forecasting of hurricane tracks. Bull. Amer. Meteor. Soc., 78, 2785-2796, https:// doi.org/10.1175/1520-0477(1997)078<2785:EFOHT>2.0.CO;2.

— , and - 1999: A perturbation method for hurricane ensemble predictions. Mon. Wea. Rev., 127, 447-470, https:/doi.org/ 10.1175/1520-0493(1999)127<0447:APMFHE > 2.0.CO;2. 